\title{
CASCADE OF SINKS
}

\author{
BY \\ CLARK ROBINSON $^{1}$
}

\begin{abstract}
ABSTRACr. In this paper it is proved that if a one-parameter family $\left\{F_{t}\right\}$ of $C^{1}$ dissipative maps in dimension two creates a new homoclinic intersection for a fixed point $P_{t}$ when the parameter $t=t_{0}$, then there is a cascade of quasi-sinks, i.e., there are parameter values $t_{n}$ converging to $t_{0}$ such that, for $t=t_{n}, F_{t}$ has a quasi-sink $A_{n}$ with each point $q$ in $A_{n}$ having period $n$. A quasi-sink $A_{n}$ for a map $F$ is a closed set such that each point $q$ in $A_{n}$ is a periodic point and $A_{n}$ is a quasi-attracting set (à la Conley), i.e., $A_{n}$ is the intersection of attracting sets $A_{n}^{j}, A_{n}=\cap_{j} A_{n}^{j}$, where each $A_{n}^{j}$ has a neighborhood $U_{n}^{j}$ such that $\bigcap\left\{F^{k}\left(U_{n}^{j}\right): k \geqslant 0\right\}=A_{n}^{j}$. Thus, the quasi-sinks $A_{n}$ are almost attracting sets made up entirely of points of period $n$. Gavrilov and Silnikov, and later Newhouse, proved this result when the new homoclinic intersection is created nondegenerately. In this case the sets $A_{n}$ are single, isolated (differential) sinks. In an earlier paper we proved the degenerate case when the homoclinic intersections are of finite order tangency (or the family is real analytic), again getting a cascade of sinks, not just quasi-sinks.
\end{abstract}

1. Definitions and statement of results. In order to state the result, several definitions are needed. For a more thorough introduction with compatible perspective see [11]. The first few definitions are given for $C^{1}$ maps (or families of maps) from a two-dimensional manifold $M$ to itself, $F: M \rightarrow M$. A point $p$ is called a periodic saddle point if $F^{k}(p)=p$ for some $k>0$ and the eigenvalues $\lambda_{u}$ and $\lambda_{s}$ of the derivative $D F^{k}(p)$ are both real, $\left|\lambda_{u}\right|>1$ and $\left|\lambda_{s}\right|<1$. A saddle point is called twisted if the eigenvalue $\lambda_{u}<-1$. (Alligood and Yorke call such points Mobius orbits.) A saddle point $p$ of period $k$ is called dissipative if $\left|\operatorname{det} D F^{k}(p)\right|<1$, so $\left|\lambda_{u} \lambda_{s}\right|<1$. The stable and unstable manifolds of a periodic saddle point $p$ are

$$
W^{s}(p, F)=\left\{q: \operatorname{distance}\left(F^{j}(p), F^{j}(q)\right) \rightarrow 0 \text { as } j \rightarrow \infty\right\}
$$

and

$$
W^{\mathrm{u}}(p, F)=\left\{q: \operatorname{distance}\left(F^{-j}(p), F^{-j}(q)\right) \rightarrow 0 \text { as } j \rightarrow \infty\right\},
$$

respectively. Note, these are the stable manifolds of the point $p$ and not the whole orbit of $p$. In the definition of $W^{\mathrm{u}}(p, F)$, if $F$ is not invertible then $F^{-j}(p)$ is a point on the periodic orbit through $p$ and $F^{-j}(q)$ means there are choices $q_{-j}$ for $F^{-j}(q)$ with $F\left(q_{-j-1}\right)=q_{-j}$ and distance $\left(F^{-j}(p), q_{-j}\right) \rightarrow 0$ as $j \rightarrow \infty$. If $p$ is a periodic saddle point, then the stable manifold theorem for noninvertible maps

Received by the editors November 21, 1983 and, in revised form, August 16, 1984. Presented to the 1983 Summer Meeting of the Society at the Special Session on Dynamical Systems II on August 11, 1983.

1980 Mathematics Subject Classification. Primary 58F12, 34D30.

${ }^{1}$ Partially supported by National Science Foundation Grant MCS 8301067. 
means there are local $C^{1}$ curves through $p, W_{\mathrm{loc}}^{\mathrm{s}}(p, F)$ and $W_{\mathrm{loc}}^{\mathrm{u}}(p, F)$. This theorem is given in many places, but it is contained in [8, Theorem 5.1] for maps, as opposed to differential equations. See [3] for other references.

A point $p$ is called a periodic sink if $F^{k}(p)=p$ for some $k>0$ and $\left|\lambda_{j}\right|<1$ for both eigenvalues $\lambda_{1}$ and $\lambda_{2}$ of $D F^{k}(p)$. A standard result shows that a sink is an attractor (asymptotically stable), i.e., there is a neighborhood $U$ of the orbit of $p$, $\mathcal{O}(p)=\left\{F^{j}(p): j \in Z\right\}$, such that $\bigcap\left\{F^{i}(U): i \geqslant 0\right\}=\mathcal{O}(p)$. With the weak hypotheses in the Theorem, the periodic points which are created are not necessarily sinks, but are sets of periodic points which form a quasi-attracting set. A closed set $A$ is called an attracting set if it is invariant and there is a neighborhood $U$ of $A$ such that $\bigcap\left\{F^{i}(U): i \geqslant 0\right\}=A$. A closed set $A$ is called a quasi-attracting set if $A$ is the intersection of attracting sets $A^{j}$. A quasi-attracting set is not necessarily attracting, as an example in Remark 3 shows. Also see [4 or 9]. A closed set $A$ is called a quasi-sink of period $n$ if $A$ is a connected quasi-attracting set and each point $q$ in $A$ is a periodic point of period $n$. In fact the quasi-sinks which are shown to exist below are either a single point or a closed curve segment.

The last few definitions and the Theorem concern a one-parameter family $\left\{F_{t}\right\}$ of $C^{1}$ maps from a two-dimensional manifold $M$ to itself, $F_{t}: M \rightarrow M$, which depends continuously on the parameter $t$ in the $C^{1}$ topology. A family $\left\{F_{t}\right\}$ is said to create (resp. destroy) homoclinic intersections at $q$ when $t=t_{0}$ for a periodic saddle point $P_{t}$ if there are $\varepsilon, k>0$ and continuously varying subarcs $\gamma_{t}^{\mathrm{s}} \subset W_{\mathrm{loc}}^{\mathrm{s}}\left(P_{t}, F_{t}\right)$ and $\gamma_{t}^{\mathrm{u}} \subset$ $W_{\mathrm{loc}}^{\mathrm{u}}\left(P_{t}, F_{t}\right)$ for $t_{0}-\varepsilon \leqslant t \leqslant t_{0}+\varepsilon$ such that

(i) $\gamma_{t}^{\mathrm{s}} \cap F_{t}^{k}\left(\gamma_{t}^{\mathrm{u}}\right)=\varnothing$ for $t_{0}-\varepsilon \leqslant t<t_{0}$ (resp. $\left.t_{0}<t \leqslant t_{0}+\varepsilon\right)$;

(ii) for $t_{0}<t \leqslant t_{0}+\varepsilon$ (resp. $\left.t_{0}-\varepsilon \leqslant t<t_{0}\right) F_{t}^{k}\left(\gamma_{t}^{\mathrm{u}}\right)$ crosses locally from one side of $\gamma_{t}^{s}$ to the other and returns to the original side;

(iii) $q \in \gamma_{t}^{\mathrm{s}} \cap \gamma_{t}^{\mathrm{u}}$ for $t=t_{0}$.

Multiple crossings are allowed in (ii), and the crossings may be $C^{\infty}$ tangent or whatever. A more precise way to state condition (ii) is that there are local coordinates $\{(x, y)\}$ near $q$ (which depend continuously on $t$ ) and parametrizations $\left\{\left(x_{t}^{\sigma}(\tau), y_{t}^{\sigma}(\tau)\right):|\tau| \leqslant \delta\right\}$ for $\sigma=s$ and $u$ of $\gamma_{t}^{\mathrm{s}}$ and $F_{t}{ }^{k}\left(\gamma_{t}^{\mathrm{u}}\right)$, respectively, such that (a) for $t=t_{0},\left(x_{t}^{\mathrm{s}}(0), y_{t}^{\mathrm{s}}(0)\right)=(0,0)=\left(x_{t}^{\mathrm{u}}(0), y_{t}^{\mathrm{u}}(0)\right)$ corresponds to $q,(\mathrm{~b}) y_{t}^{\mathrm{s}}(\tau) \equiv 0$, and (c) for $t_{0}<t \leqslant t_{0}+\varepsilon, y_{t}^{\mathrm{u}}(-\delta)$ and $y_{t}^{\mathrm{u}}(\delta)$ are of one sign and $y_{t}^{\mathrm{u}}\left(\tau^{\prime}\right)$ is of another sign for some $\left|\tau^{\prime}\right|<\delta$, where $\tau^{\prime}$ may depend on $t$. The family nondegenerately creates the homoclinic intersection if the family is $C^{2}$ and, in addition to (i) and (ii), satisfies

(iii) for $t=t_{0}, \gamma_{t}^{\mathrm{s}}$ and $\gamma_{t}^{\mathrm{u}}$ have an intersection of order two at $q$ (tangency of order one), i.e., for $t=t_{0}$,

$$
y_{t}^{\mathrm{u}}(0)=0=\frac{d}{d \tau} y_{t}^{\mathrm{u}}(0) \quad \text { but } \quad \frac{d^{2}}{d \tau^{2}} y_{t}^{\mathrm{u}}(0) \neq 0 ;
$$

(iv) if $y_{t}^{*}$ is the extreme value of $y_{t}^{\mathrm{u}}(\tau)$ for $-\delta \leqslant \tau \leqslant \delta$ then $d y^{*} / d t \neq 0$ at $t=t_{0}$.

Note, when the family nondegenerately creates homoclinic intersections, then for $t>t_{0}$ there are two new transverse intersections and a Smale horseshoe.

A family $\left\{F_{t}\right\}$ has a cascade of quasi-sinks (resp. cascade of sinks) if there is a sequence of parameter values $t_{n}$ converging to $t_{0}$ such that, for $t=t_{n}, F_{t}$ has a quasi-sink $p_{n}$ (resp. a sink) of period $b_{n}$ and $b_{n}$ goes to infinity as $n$ goes to infinity. In 
the Theorem, where the cascade is caused by the creation of a homoclinic intersection for a periodic saddle point $P_{t}$ of period $a$, then $b_{n}=n a+k$, where $F_{t}^{k}\left(W_{\text {loc }}^{\mathrm{u}}\left(P_{t}, F_{t}\right)\right)$ intersects $W_{\mathrm{loc}}^{\mathrm{s}}\left(P_{t}, F_{t}\right)$. In particular if $P_{t}$ is a fixed point then $b_{n}=n$ and all sufficiently large periods are attained. The Theorem can now be stated.

THEOREM. Let $\left\{F_{t}\right\}$ be a one-parameter family of $C^{1}$ maps of a two-dimensional manifold $M$ which creates a homoclinic intersection at $q$ when $t=t_{0}$ for a dissipative periodic saddle point $P_{t}$ of period a. Then $\left\{F_{t}\right\}$ has a cascade of quasi-sinks $A_{n}$ of period $b_{n}$ with the following properties.

(1) If $P_{t}$ is a family of fixed points then the periods $b_{n}=n$ for $n$ greater than some $N$. In general, $b_{n}=a n+k$, where $a$ is the period of $P_{t}$ and $F_{t}^{k}\left(W_{\mathrm{loc}}^{\mathrm{u}}\left(P_{t}, F_{t}\right)\right)$ intersects $W_{\mathrm{loc}}^{\mathrm{s}}\left(P_{t}, F_{t}\right)$.

(2) If $F_{t}^{a}$ preserves the orientation of $W^{\mathrm{s}}\left(P_{t}, F_{t}\right)$ and $W^{\mathrm{u}}\left(P_{t}, F_{t}\right)$ then the parameter values $t_{n}$ converge to $t_{0}$ from one side. In some cases $t_{n}>t_{0}$ and in other cases $t_{n}<t_{0}$. If $F_{t}^{a}$ reverses either or both of the orientations, then the $t_{n}$ for odd $n$ converge to $t_{0}$ from one side and those for even $n$ converge from the other side.

(3) As $n$ goes to infinity, the quasi-sinks $A_{n}$ converge to an invariant subset of the orbit $F_{t_{0}}$ of $\gamma_{t_{0}}^{\mathrm{u}} \cap \gamma_{t_{0}}^{\mathrm{s}}, \mathcal{O}\left(\gamma_{t_{0}}^{\mathrm{u}} \cap \gamma_{t_{0}}^{\mathrm{s}}\right)$.

The proof is contained in $\S 2-4$. $\S 2$ considers the case where, for each $t, P_{t}$ is a fixed point and $F_{t}$ preserves the orientations of $W^{\mathrm{s}}\left(P_{t}, F_{t}\right)$ and $W^{\mathrm{u}}\left(P_{t}, F_{t}\right) . \S 3$ indicates the necessary changes when $F_{t}$ reverses one of the orientations. Finally, $\$ 4$ indicates the changes when $P_{t}$ is a periodic point.

REMARK 1. As mentioned in the abstract, it has been previously proved that $\left\{F_{t}\right\}$ has a cascade of sinks (not just quasi-sinks) whenever the family nondegenerately creates a homoclinic intersection, or when it degenerately creates a homoclinic intersection of finite order. The nondegenerate case was proved by Gavrilov and Silnikov [6] and was also contained in the work of Newhouse [10 or 11]. The finite-order case was proved in our earlier paper [12] and always applies when the maps are real analytic. The present proof uses a lemma from this latter paper, which states that whenever a family creates a twisted periodic saddle point then somewhere before there is a periodic sink. However, in this paper an index argument is used rather than the analytic estimate, which shows a twisted periodic saddle is created whenever a finite-order homoclinic intersection is created. The present theorem is also related to, but different than, the recent result of Alligood and Yorke [1]. They assume a horseshoe is created as the parameter varies, which is true if the family nondegenerately creates homoclinic intersections, but is probably not true if it occurs degenerately. Their conclusion is much stronger than the above Theorem: rather than just one cascade of sinks, they show, via period doubling, there are many cascades of sinks. Thus, their assumptions and conclusions are both stronger. The paper by Aronson et al. [2] contains a numerical study where a homoclinic intersection is created for a noninvertible map. Thus, the Theorem proves the sinks they observe are actually there. For a map with singularities as simple as they study, there are parameter values with infinitely many sinks as in [10 or 12]. It is not clear what general assumptions insure infinitely many sinks for a noninvertible map. 


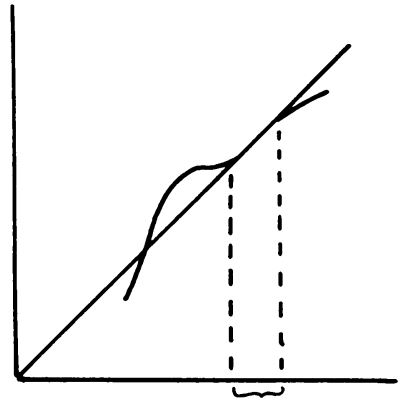

(a)
A

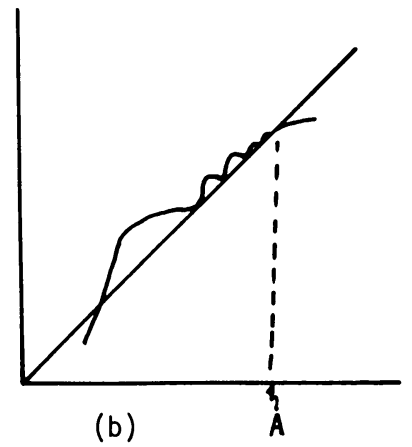

(b)

FIGURE 1

REMARK 2. The quasi-sinks which are not sinks have a lot of structure, and their basins of attraction are much like the basins of a sink. Let $A_{n}$ be such a quasi-sink of period $n$. The points of $A_{n}$ are center-stable periodic points with eigenvalue $\lambda_{1}=1$ and $\left|\lambda_{2}\right|<1$. The Center Manifold Theorem implies that $A_{n}$ lies on a $C^{1}$ curve segment $\gamma_{n}$, which is strongly contracting normally to $\gamma_{n}$. Moreover, the proof shows that $A_{n}=\bigcap_{j} A_{n}^{j}$, where $A_{n}^{j}$ is a sequence of decreasing curve segments in $\gamma_{n}$ with endpoints of period $n$ and weakly attracting in $\gamma_{n}$. (The contraction is higher order because $\lambda_{1}=1$.) By minimality, $A_{n}$ is either a point or a curve segment made up entirely of points of period $n$. If $A_{n}$ is a curve segment then, since it is normally attracting, its stable manifold (or basin of attraction) will contain an open subset, so it is much like the basin of a sink. If $A_{n}$ is a point then the points asymptotic to $A_{n}$ might not contain an open set. In any case the stable manifolds of $A_{n}^{j}$ contain an open set. With a given level of precision of iteration (on a computer for example), the set $A_{n}$ would not be distinguished from $A_{n}^{j}$ with $j$ large, so the basin of attraction of $A_{n}^{j}$ would be the effective basin of attraction of $A_{n}$. Thus, the quasi-sink would be visible.

REMARK 3. To give some understanding of how quasi-sinks occur, as opposed to sinks, consider a family of maps of the interval $f_{t}: I \rightarrow I$. Assume, for $t<0$, that $f_{t}$ has no fixed points and, for $t=0$, that $f_{t}$ has a degenerate fixed point. For all $t>0$ it is possible that $f_{t}$ has two intervals of fixed points, one of which is attracting (see Figure 1(a)). It is also possible that, for $t>0, f_{t}$ has a single point as a quasi-sink, but it is only quasi-attracting and not attracting, e.g., the quasi-sink could be the limit of a countable sequence of fixed points (saddle nodes). See Figure 1(b). For a discussion of such quasi-attracting sets see the papers by Conley [4] or Hurley [9].

2. Proof for orientation-preserving fixed saddle point. In this section it is assumed that the homoclinic intersection is created at $q_{0}$ for a fixed saddle point $P_{t}$. Also it is assumed that $F_{t}$ preserves the orientation of $W^{\mathrm{u}}\left(P_{t}, F_{t}\right)$ and $W^{\mathrm{s}}\left(P_{t}, F_{t}\right)$. For simplicity of argument, $F_{t}$ is assumed to be a $C^{2}$ local diffeomorphism, so there exist $C^{1}$ coordinates $(x, y)$ on a neighborhood $U$ of $P_{t}$ in which $F_{t}$ is linear, $F_{t}(x, y)=$ $(\mu x, \lambda y)$ with $0<\mu<1$ and $1<\lambda$. (If $F_{t}$ cannot be linearized, e.g., if $F_{t}$ is not invertible at $P_{t}$, then it is necessary to use $C^{1}$ coordinates where $F_{t}$ is almost linear 

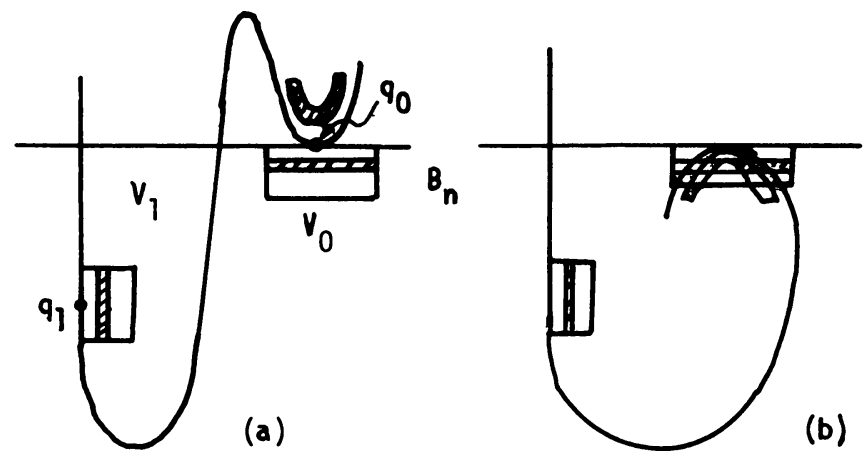

FIGURE 2

and the local stable and unstable manifolds are the coordinate axes. See [12, Appendix 5.4] for use of such coordinates.) The point $P_{t}$ is dissipative, so $\lambda \mu<1$ or $\mu<\lambda^{-1}$. By looking along the orbit of $q_{0}, q_{0}=\left(x_{0}, 0\right)$ can be assumed to lie on the local stable manifold of $P_{t}$ in $U$. Also, by taking $k$ large enough there is a $q_{1}=\left(0, y_{1}\right)$ on the local unstable manifold of $P_{t}$ in $U$ with $F_{t_{0}}^{k}\left(q_{1}\right)=q_{0}$.

Next, boxes $B_{n}$ are chosen near $q_{0}$ lying in the quadrant abutting $q_{0}$ and $q_{1}$. Depending on the way the homoclinic intersection is formed, the boxes $F_{t}^{n}\left(B_{n}\right)$ are pulled off $B_{n}$ for either $t>t_{0}$ or $t<t_{0}$. See Figures 2(a) and 2(b) for examples of the respective cases. It is proved below that as $F_{t}{ }^{n}\left(B_{n}\right)$ is pulled off $B_{n}$, there has to be some value of $t$ in which there is a quasi-sink. Take $\delta_{1}^{\mathrm{u}}, \delta_{1}^{\mathrm{s}}, \delta_{0}^{\mathrm{u}}, \delta_{0}^{\mathrm{s}}>0$ and form

$$
\begin{aligned}
& V_{0} \equiv\left\{(x, y):\left|x-x_{0}\right| \leqslant \delta_{0}^{s}, 0 \leqslant\left(\operatorname{sign} y_{1}\right) y \leqslant \delta_{0}^{u}\right\}, \\
& V_{1} \equiv\left\{(x, y):\left|y-y_{1}\right| \leqslant \delta_{1}^{u}, 0 \leqslant\left(\operatorname{sign} x_{0}\right) x \leqslant \delta_{1}^{s}\right\} .
\end{aligned}
$$

The value $\delta_{0}^{\mathrm{s}}$ has to be large enough so that $\gamma_{t_{0}}^{\mathrm{s}} \cap \gamma_{t_{0}}^{\mathrm{u}}$ is contained in the boundary of $V_{0}$. In fact, $\delta_{0}^{\mathrm{s}}$ and $\gamma_{t}^{\mathrm{s}}$ are chosen such that $\gamma_{t}^{\mathrm{s}} \subset$ boundary $V_{0}$. Similarly, $\delta_{1}^{\mathrm{u}}$ and $\gamma_{t}^{\mathrm{u}}$ are chosen so that $F_{t}^{-k}\left(\gamma_{t}^{\mathrm{u}}\right) \subset$ boundary $V_{1}$. For $N$ large enough, $n \geqslant N$, and $m=n-k$, the box $B_{n}$ is defined by

$$
\begin{aligned}
B_{n} & \equiv \operatorname{component}\left(V_{0} \cap F_{t}^{-m}\left(V_{1}\right)\right) \\
& =\left\{(x, y):\left|x-x_{0}\right| \leqslant \delta_{1}^{\mathrm{s}},\left|y-\lambda^{-m} y_{1}\right|=\lambda^{-m} \delta_{1}^{\mathrm{u}}\right\} .
\end{aligned}
$$

Thus, $B_{n}$ is a horizontal strip near $q_{0}$. The component is chosen to be the first intersection with $V_{0}$ along $F_{t}^{-m}\left(V_{1}\right)$. Then $F_{t}^{m}\left(B_{n}\right)$ is a vertical strip near $q_{1}$, and $F_{t}^{n}\left(B_{n}\right)=F_{t}^{k} \circ F_{t}^{m}(B)$ is a thin nonlinear box near $q_{0}$ which is parallel to $\gamma_{t}^{\mathrm{u}} \subset$ $W^{\mathrm{u}}\left(P_{t}, F_{t}\right)$.

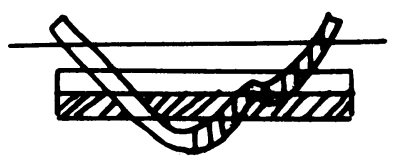

(a)

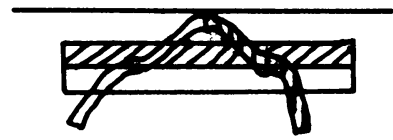

(b)

FiguRE 3 
For $N$ large enough there is an $\varepsilon>0$ such that

(1) $F_{t}^{n}\left(B_{n}\right) \cap B_{n}=\varnothing$ for either (a) $t=t_{0}$ or, respectively, (b) $t=t_{0}-\varepsilon$;

(2) for either (a) $t=t_{0}+\varepsilon$ or, respectively, (b) $t=t_{0}, F_{t}^{n}\left(B_{n}\right)$ crosses $B_{n}$

(i) from top to bottom and back again as in Figure 3(a), or

(ii) from bottom to top and back again as in Figure 3(b);

(3) $F_{t}^{n}\left(B_{n}\right)$ never intersects the sides of $B_{n}$ for $t_{0}-\varepsilon \leqslant t \leqslant t_{0}+\varepsilon$; and

(4) the images of the top and bottom of $B_{n}$ by $F_{t}^{n}$ never intersect $B_{n}$ for $t_{0}-\varepsilon \leqslant t \leqslant t_{0}+\varepsilon$.

The crossings are not necessarily monotone, and there can be several crossings. In any case there is a substrip $B_{n}^{\prime}$ of $B_{n}$,

$$
B_{n}^{\prime}=\left\{(x, y):\left|x-x_{0}\right| \leqslant \delta_{1}^{\mathrm{s}}, y_{n}^{\prime} \leqslant y \leqslant y_{n}^{\prime \prime}\right\} \subset B_{n},
$$

such that $F_{t}^{n}\left(x, y_{n}^{\prime}\right)$ lies above $B_{n}$ and $F_{t}^{n}\left(x, y_{n}^{\prime \prime}\right)$ lies below $B_{n}$ for (a) $t=t_{0}+\varepsilon$ or, respectively, (b) $t=t_{0}$. Since $F_{t}^{n}$ has no fixed points on the boundary of $B_{n}^{\prime}$, it easily follows that the Lefschetz index of $F_{t}^{n}$ relative to $B_{n}^{\prime}$ is $+1, I\left(B_{n}^{\prime}, F_{t}^{n}\right)=I\left(B_{n}^{\prime}\right)=1$. (See [7 or 5] for a discussion of the index. It is locally much like the index of an equilibrium point for a vector field or differential equation as discussed in many differential equations books.) If the fixed points of $F_{t}^{n}$ in $B_{n}^{\prime}$ are isolated, then the sum of their indices is 1 . In any case since $P_{t}$ is dissipative, $F_{t}^{n}$ restricted to $B_{n}$ decreases area, so the possible fixed points of $F_{t}^{n}$ in $B_{n}^{\prime}$ are as follows:

\begin{tabular}{llc}
\multicolumn{1}{c}{ Type } & \multicolumn{1}{c}{ Eigenvalues } & Index \\
weak twisted saddle & $\lambda_{1} \leqslant-1,\left|\lambda_{2}\right|<1$ & 1 \\
sink & $\left|\lambda_{1}\right|<1,\left|\lambda_{2}\right|<1$ & 1 \\
center-stable & $\lambda_{1}=1,\left|\lambda_{2}\right|<1$ & $-1,0$, or 1 \\
saddle & $\lambda_{1}>1,\left|\lambda_{2}\right|<1$ & -1
\end{tabular}

All the fixed points, except possibly the center-stable points, are isolated. If there are no center-stable points then for $t=t_{0}+\varepsilon$ or $t=t_{0}$,

$$
1=I\left(B_{n}^{\prime}, F_{t}^{n}\right)=\sum\left\{I\left(q, F_{t}^{n}\right): q \text { is a fixed point of } F_{t}^{n}\right\} .
$$

Thus, there must be either a sink or a weak twisted saddle point. (The adjective weak refers to the fact that $\lambda_{1}=-1$ is allowed.) If there is a sink, then the proof is finished. Otherwise, there is a weak twisted periodic saddle point. As the parameter $t$ varies from $t_{0}+\varepsilon$ to $t_{0}$ (respectively, $t_{0}$ to $t_{0}-\varepsilon$ ), the twisted saddle fixed point of $F_{t}^{n}$ in $B_{n}$ disappears without crossing the boundary of $B_{n}$ because of properties (3) and (4) above. By applying [12, Proposition 3.3] it follows that, for some intermediate parameter value $t=t_{n}$, there is a sink.

If $F_{t}^{n}$ has some center-stable fixed points, then the following argument shows there must be a quasi-sink for some parameter value. Let $S$ be the set of center-stable fixed points of $F_{t}^{n}$ in $B_{n}^{\prime}$. For any $q$ in $S$ one of the eigenvalues $\left|\lambda_{2}\right|<1$, so by the Center Manifold Theorem [3] there is a neighborhood $W$ of $q$ and a $C^{1}$ curve $C$ such that all the fixed points of $F_{t}^{n}$ in $W$ lie on $C$. By extending the curves when the neighborhoods overlap, the endpoints of $C$ can be chosen off $S$. The extended curves $C$ cannot be closed curves because they are invariant and $F_{t}^{n}$ decreases area and the 
interior would be invariant. The endpoints of the curves $C$ either (i) both move inward so $I\left(C, F_{t}{ }^{n}\right)=1$, (ii) both move outward so $I\left(C, F_{t}{ }^{n}\right)=-1$, or one end moves inward and the other outward so $I\left(C, F_{t}^{n}\right)=0$. The set $S$ can be covered by a finite number of neighborhoods $W_{i}$ with corresponding curve segments $C_{i}$. Then

$$
\begin{aligned}
1 & =I\left(B_{n}^{\prime}, F_{t}^{n}\right) \\
& =\sum I\left(C_{i}, F_{t}^{n}\right)+\sum\left\{I\left(q, F_{t}^{n}\right): q=F_{t}^{n}(q) \text { is not center-stable }\right\} .
\end{aligned}
$$

Since the sum is 1 , one of the terms must be 1 . If there is a sink or twisted saddle, then the argument is similar to the above. Otherwise, there is a curve segment $C_{i}$ with index 1. If $C_{i} \cap S$ is an interval of fixed points, then it is attracting, and so a quasi-sink. Otherwise, there are a finite or a countable number of open subcurves $\left\{J_{i j}\right\}$ in $C_{i}$ in which the points are moved by $F_{t}{ }^{n}$. For any such $J_{i j}$ the points are all moved in one direction on $C_{i}$. Then one-half of $C_{i}-J_{i j}$ is an attracting set, $A_{n}^{j}$. By picking the attracting sets $A_{n}^{j}$ from $A_{n}^{j-1}-J_{i j}$ sequentially, $A_{n}=\bigcap_{j} A_{n}^{j}$ is a quasi-sink. By construction, the set $A_{n}$ is closed and connected, so it is either a point or a curve segment, and it is made up entirely of points of period $n$.

Property 3 of the Theorem follows because the boxes $B_{n}$ can be made to converge to $\gamma_{t_{0}}^{\mathrm{u}} \cap \gamma_{t_{0}}^{\mathrm{s}}$ by letting $\delta_{0}^{\mathrm{s}}$ depend on $n$.

3. Orientation reversing case. In this section $P_{t}$ is still assumed to be a fixed point of $F_{t}$. If $F_{t}$ preserves the orientation of $W^{\mathrm{s}}\left(P_{t}, F_{t}\right)$, let $V_{1}$ be as before. If it reverses orientation of $W^{\mathrm{s}}\left(P_{t}, F_{t}\right)$, let

$$
V_{1} \equiv\left\{(x, y):\left|y-y_{1}\right| \leqslant \delta_{1}^{u},|x| \leqslant \delta_{1}^{\mathrm{s}}\right\} .
$$

Similarly, if $F_{t}$ reverses the orientation of $W^{\mathrm{u}}\left(P_{t}, F_{t}\right)$, change the definition of $V_{0}$ to

$$
V_{0} \equiv\left\{(x, y):\left|x-x_{0}\right| \leqslant \delta_{0}^{\mathrm{s}},|y| \leqslant \delta_{0}^{\mathrm{u}}\right\} .
$$

Let $B_{n} \equiv$ component $\left(V_{0} \cap F_{t}^{-m}\left(V_{1}\right)\right)$, where $m=n-k$. If $F_{t}$ preserves the orientation of $W^{\mathrm{u}}\left(P_{t}, F_{t}\right)$, then $B_{n}$ is on the same side of $W^{\mathrm{u}}\left(P_{t}, F_{t}\right)$; if it reverses orientation, then $B_{n}$ is on different sides depending on the parity of $n$. In the same

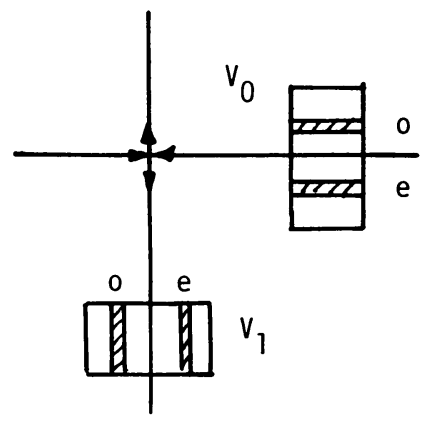

FIGURE 4 
way, $F_{t}^{m}\left(B_{n}\right)$ is either on one side of $W^{\mathrm{u}}\left(P_{t}, F_{t}\right)$ or both sides depending on whether or not $F_{t}$ preserves the orientation of $W^{\mathrm{s}}\left(P_{t}, F_{t}\right)$. The same applies to $F_{t}^{n}\left(B_{n}\right)$ and the sides of $\gamma_{t}^{\mathrm{u}}$.

The rest of the proof is similar to $\S 2$. If only one of the orientations is reversed, it is not hard to see that the $t_{n}$ which gives a quasi-sink of period $n$ will be on different sides of $t_{0}$ depending on the parity of $n$. The same statement is true if both orientations are reversed, but more care is needed in the argument. In the case depicted in Figure 5(a-i,ii), it is clear the $t_{n}$ are on opposite sides of $t_{0}$ depending on the parity of $n$. In the case depicted in Figure 5(b-i), it is harder. Because for $t=t_{0}$ the distance from $F_{t}^{n}\left(B_{n}\right)$ to $\gamma_{t}^{\mathrm{u}}$ is proportional to $\mu^{m}$, the distance from $B_{n}$ to $\gamma_{t}^{\mathrm{s}}$ is proportional to $\lambda^{-m}$, and $\mu^{m}<\lambda^{-m}, F_{t}^{n}\left(B_{n}\right)$ and $B_{n}$ intersect for $n$ odd and do not intersect for $n$ even. Thus, in this case the quasi-sinks for $n$ odd occur for $t_{n}<t_{0}$ and for $n$ even for $t_{n}>t_{0}$. All other cases are similar to one of these two. This completes the argument.

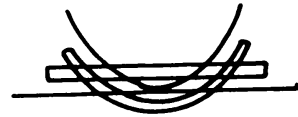

$a-i$ odd

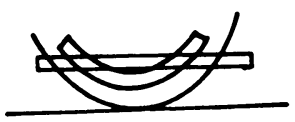

$b-i$ odd

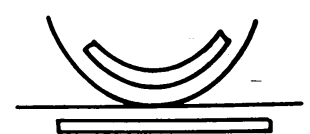

$a-i j$ even

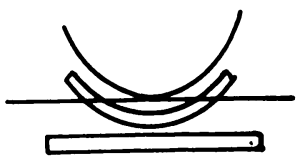

$b-i j$ even

FigURE 5

4. Periodic case. When $P_{t}$ has period $a, k$ is chosen so that $F_{t}^{k}\left(q_{1}\right)=q_{0}$ with $q_{1} \in W_{\mathrm{loc}}^{\mathrm{u}}\left(P_{t}, F_{t}\right)$. Note, $q_{1}$ is on the local unstable manifold for $P_{t}$ and not another point of the orbit of $P_{t}$. The sets $V_{0}$ and $V_{1}$ are constructed near $q_{0}$ and $q_{1}$, respectively, as before. Let $B_{n}=\operatorname{component}\left(V_{0} \cap F_{t}^{-n a}\left(V_{1}\right)\right)$. As before, for $b=b_{n}$ $=n a+k, F_{t}^{b}$ has a quasi-sink in $B_{n}$ for a suitable chosen parameter value $t$.

\section{REFERENCES}

1. K. Alligood and J. Yorke, Cascades of period-doubling bifurcations: a prerequisite for horseshoes, Bull. Amer. Math. Soc. (N. S.) 9 (1983), 319-322.

2. D. G. Aronson, M. A. Chory, G. R. Hall and R. P. McGehee, Bifurcation from an invariant circle for two-parameter families of the plane, Comm. Math. Phys. 83 (1982), 303-354.

3. J. Carr, Applications of centre manifold theory, Springer-Verlag, 1981.

4. C. Conley, Isolated invariant sets and the Morse index, CBMS Regional Conf. Ser. in Math., no. 38, Amer. Math. Soc., Providence, R.I., 1978.

5. J. Franks, Homology and dynamical systems, CBMS Regional Conf. Ser. in Math., no. 49, Amer. Math. Soc., Providence, R.I., 1982.

6. N. K. Gavrilov and L. P. Silnikov, On the three dimensional dynamical system close to a system with a structurally unstable homoclinic curve. I, II, Math. USSR Sb. 17 (1972), 467-485; ibid. 19 (1973), 139-156. 
7. V. Guillemin and A. Pollack, Differential topology, Prentice-Hall, Englewood Cliffs, N.J., 1974.

8. M. Hirsch, C. Pugh and M. Shub, Invariant manifolds, Lecture Notes in Math., vol. 583, Springer-Verlag, 1977.

9. M. Hurley, Attractors: persistence and density of their basins, Trans. Amer. Math. Soc. 269 (1982), 247-271.

10. S. Newhouse, The abundance of wild hyperbolic sets and nonsmooth stable sets for diffeomorphisms, Inst. Hautes. Études Sci. Publ. Math. 50 (1979), 101-151.

11. __ Lectures on dynamical systems, Progress in Math. vol. 8, Birkhäuser, 1980, pp. 1-114.

12. C. Robinson, Bifurcation to infinitely many sinks, Comm. Math. Phys. 90 (1983), 433-459.

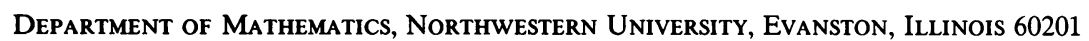

